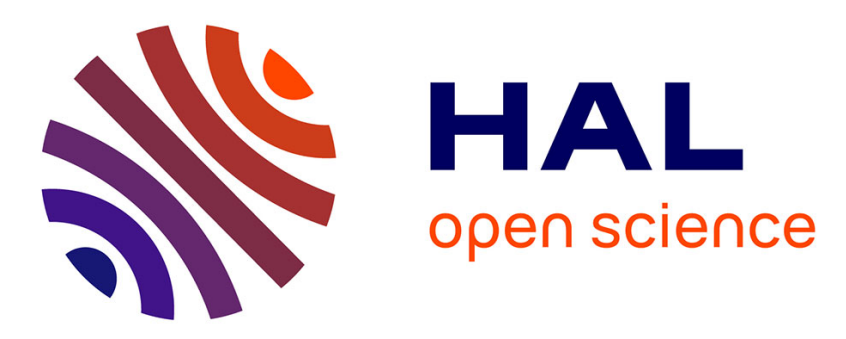

\title{
Molecular dynamics simulations of high energy cascade in ordered alloys: Defect production and subcascade division
}

Laurence Luneville, Jean-Paul Crocombette, Laurent van Brutzel, David Simeone, Laurence Lunéville

\section{To cite this version:}

Laurence Luneville, Jean-Paul Crocombette, Laurent van Brutzel, David Simeone, Laurence Lunéville. Molecular dynamics simulations of high energy cascade in ordered alloys: Defect production and subcascade division. Journal of Nuclear Materials, 2016, 474, pp.134-142. 10.1016/j.jnucmat.2016.03.020 . hal-02429493

\section{HAL Id: hal-02429493 \\ https://hal.science/hal-02429493}

Submitted on 24 Mar 2020

HAL is a multi-disciplinary open access archive for the deposit and dissemination of scientific research documents, whether they are published or not. The documents may come from teaching and research institutions in France or abroad, or from public or private research centers.
L'archive ouverte pluridisciplinaire HAL, est destinée au dépôt et à la diffusion de documents scientifiques de niveau recherche, publiés ou non, émanant des établissements d'enseignement et de recherche français ou étrangers, des laboratoires publics ou privés. 


\title{
Molecular dynamics simulations of high energy cascade in ordered alloys: Defect production and subcascade division
}

\author{
Jean-Paul Crocombette ${ }^{\text {a, * }}$, Laurent Van Brutzel ${ }^{b}$, David Simeone ${ }^{c}$, Laurence Luneville ${ }^{d}$ \\ ${ }^{a}$ CEA, DEN, Service de Recherches de Métallurgie Physique, UPSay, F-91191 Gif-sur-Yvette, France

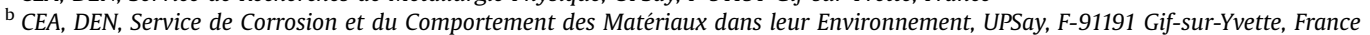 \\ ${ }^{c}$ CEA, DEN, Service de Recherches de Métallurgie Appliqué, Matériaux Fonctionnels pour l'Energie, CNRS-CEA-ECP, UPSay, F-91191 Gif-sur-Yvette, France \\ d CEA, DEN, Service d'Etudes des Réacteurs et de Mathématiques Appliquées, Matériaux Fonctionnels pour l'Energie, CNRS-CEA-ECP, UPSay, F-91191 Gif-sur- \\ Yvette, France
}

\section{G R A P H I C A L A B S T R A C T}

Molecular Dynamics simulations of cascade in ordered alloys show that defect production in such

(1) monelemental solids

therion of

subcascades does not hold in general for alloys.

OPKA

$\mathrm{OPKA}$ in $\mathrm{OO}_{2}$
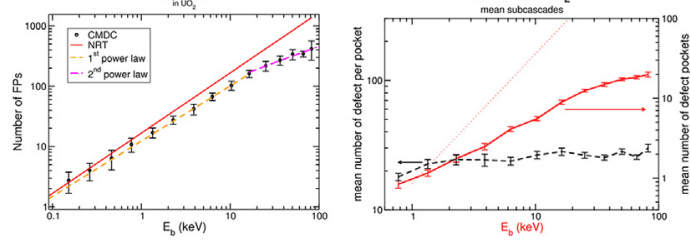

\section{A B S T R A C T}

Displacement cascades have been calculated in two ordered alloys $\left(\mathrm{Ni}_{3} \mathrm{Al}\right.$ and $\left.\mathrm{UO}_{2}\right)$ in the molecular dynamics framework using the $\mathrm{CMDC}$ (Cell Molecular Dynamics for Cascade) code (J.-P. Crocombette and T. Jourdan, Nucl. Instrum. Meth. B 352, 9 (2015)) for energies ranking between 0.1 and $580 \mathrm{keV}$. The defect production has been compared to the prediction of the NRT (Norgett, Robinson and Torrens) standard. One observes a decrease with energy of the number of defects compared to the NRT prediction at intermediate energies but, unlike what is commonly observed in elemental solids, the number of produced defects does not always turn to a linear variation with ballistic energy at high energies. The fragmentation of the cascade into subcascades has been studied through the analysis of surviving defect pockets. It appears that the common knowledge equivalence of linearity of defect production and sub-cascades division does not hold in general for alloys. We calculate the average number of subcascades and average number of defects per subcascades as a function of ballistic energy. We find an unexpected variety of behaviors for these two average quantities above the threshold for subcascade formation.

\footnotetext{
* Corresponding author.

E-mail address: jpcrocombette@cea.fr (J.-P. Crocombette).
} 


\section{Introduction}

In a nuclear environment, materials are submitted to several severe solicitations among which neutron irradiation producing transmutations and atomic displacements. In order to compare different neutron spectra as well as to mimic with ion beams the atomic displacements induced by neutrons, one needs a unit of measurement for the damage sustained by the material. Such damage comes mainly from the displacement cascades induced by ballistic collisions between fast moving particles (neutrons or ions) with atoms in the material. Kinchin and Pease [1] estimated the number of displaced atoms by a PKA (Primary Knock-on Atom) in terms of its energy for ballistic collisions, hereafter referred to as the ballistic energy $\left(E_{b}\right)$. This energy has sometimes been labelled as damage energy. Both terms refer to exactly the same thing. We prefer to use ballistic energy as it more clearly connects to the ballistic loses of the projectiles. For energies larger than twice the threshold displacement energy $\left(E_{d}\right)$ in the material (which is defined as the kinetic energy needed to displace permanently an atom from its original lattice site) the number of displaced atoms follows a linear form:

$N_{\text {disp. }}^{K P}=\frac{E_{b}}{2 E_{d}}$

It was very early recognized that what is really interesting is not the number of displacements but rather the number of defects created, and that these two numbers are not equivalent. Norgett, Robinson and Torrens (NRT) [2], proposed a simple rescaling by a factor 0.8 of the KP formula for $E_{b}$ larger than $2 E_{d}$ to obtain the number of created Frenkel (interstitial-vacancy) pairs (FPs) in a material:

$N_{\mathrm{FP}}^{N R T}=0.4 \frac{E_{b}}{E_{d}}$

This "NRT" formula is the base of an ASTM standard [3] for the calculation of the number of created FPs. Dividing $N_{\mathrm{FP}}^{N R T}$ by the number of atoms in the material gives a measure of damage named "dpa", which stands for "displacement per atom". In spite of this name NRT formula and dpa really aim to estimate the number of surviving FPs defects.

It has long been acknowledged, both experimentally (e.g. Ref. [4]) and from Molecular Dynamics (MD) simulations ${ }^{5} 6$ that the NRT formula overestimates the amount of created defects. MD simulations have shown that this overestimation comes from the partial recrystallization that takes place during the heat spike of the cascade.

One then usually defines an efficiency factor $(\chi)$ as the ratio of the actual number of created defects to the NRT prediction. The efficiency in some simple metals, especially iron [7,8], has been the subject of detailed studies. It has been found that the number of defects evolves as a power law with the ballistic energy then switches for high ballistic energies to a linear dependence with an efficiency factor equal to about 1/3. Following Averback [5] and Stoller [7], one commonly relates this saturation at high energy cascades of $\chi$ to the split into disconnected subcascades.

In this paper we study the defect creation by displacement cascades in ordered alloys at large energies. We perform cascade simulations using MD on two ordered alloys: $\mathrm{UO}_{2}$ and $\mathrm{Ni}_{3} \mathrm{Al}$. We calculate the number of created defects and discuss its evolution with ballistic energy, checking if the evolution of $\chi$ mentioned above is observed in alloys, namely first a power law decrease then a saturation to a constant value. The obtained results lead us to revisit the assumed relationship between the apparition of subcascades and the linearity of defect production.

Several MD calculations of cascades in ordered alloys already exist in literature [9-15]. However, most of them are limited to low energy PKA. Moreover, they consider cascades created by the heavy elements of the alloy (e.g. $\mathrm{U}$ in $\mathrm{UO}_{2}[12,13]$ or $\mathrm{Ni}$ in $\mathrm{Ni}_{3} \mathrm{Al}^{11,15}$ ). This choice probably relates to the difficulty, with standard MD, of calculating cascades induced by light elements, because of the very large systems that such calculations require. In both $\mathrm{UO}_{2}$ and $\mathrm{Ni}_{3} \mathrm{Al}$ the number of defects is known to follow a power law at lower ballistic energies [11-13].

In the present paper we treat on equal footage PKAs of both elements of the alloys. The present calculations use the recently developed Cell Molecular Dynamics for Cascade (CMDC) [16] code, which circumvents the problem of intractably large systems.

The paper is organized as follows. The next part deals with methods and technicalities of the simulations. The following part discusses the results pertaining to the number of created defects. Part IV and V tackle the formation of subcascades, their average number and size and the possible connection with the linearity of defect production.

\section{Methods}

\subsection{Cell molecular dynamics for cascade}

Cascade simulations have been performed with the CMDC code, which is a MD code designed specifically to accelerate the calculations of displacement cascades. CMDC is based on the observation that, in standard MD calculations, many parts of the system do not take part of the cascade. Since the trajectories of the cascade are unpredictable, such large systems are necessary to embrace the full development of the cascade. The core principle of CMDC is then to perform a regular MD simulation but just where and when necessary to properly describe the cascade unfolding. We only briefly present the CMDC code as it has been described in details elsewhere [16]. The first point of the code is to build the MD box during the unfolding of the cascade. Crystalline cells are added and removed on the fly from the calculation based on local kinetic energy criteria. When the maximal or the average kinetic energy of the atoms in a cell exceeds given thresholds, the neighboring cells are turned on. The atoms included in these cells are then given random initial velocities and displacements corresponding to the temperature of interest. In the present work, only room temperature has been considered but, in principle, any temperature can be considered. Symmetrically, cells are turned off from the simulation when kinetic energy in all their neighboring cells becomes lower than a given threshold. When expressed in kinetic energy, the turning on and turning off criteria are of the order $0.1 \mathrm{eV}$ per atom at most.

The choice of temperature thresholds proceeds as follows. When temperature thresholds are fixed at too low values, i.e. too close from the initial temperature, the calculations diverges in the sense that cells continuously add up to the simulation. Indeed, because of their small number of atoms, the thermal fluctuations in the cells are substantial so that too low thresholds are reached simply by thermal fluctuations even in the absence of kinetic energy input by the cascades. These low barriers for the thresholds depend on the number of atoms in the cells which in turn depend on the range of interaction potentials. At the opposite, above a certain limit, when temperatures thresholds are increased, one observes that the number of defects produced on average by cascades increases with the thresholds. Indeed, increasing turning-on temperature thresholds confines the cascades in smaller and smaller volumes to accommodate the same energy input, so that the disorder increases. Symmetrically increasing the turning-off temperature thresholds implicitly allows for less and less time for crystal recovery at the end of the cascades. In between these low and high limits of temperature thresholds, one observes that the numbers of defects produced are statistically equal. In practice these limits are found by a trial and error procedure on medium energy cascades (a few tens of keV). The actual thresholds should then be chosen in the interval where the number of produced 
defects does not vary, preferably, for the sake of calculation speed, close the upper limit of the interval. The procedure should be redone if one is interested in another initial temperature.

Due to the abrupt freezing of atoms in turned off cells, the structure at the end of the simulation may be trapped in an unstable configuration. To allow for some relaxations to take place, all the atomic positions in the cells that were active at some point of the cascade unfolding are eventually relaxed at the end of the simulation with a so-called fast quenching algorithm with asynchronous turning off of cells as in the cascade simulation. Mechanically unstable defects are thus eliminated.

The second point of the code is to use a space variable time step for the simulation, applying a different time step for each active cell. While cells are active, atoms move according to a standard velocity Verlet algorithm.

Thirdly, CMDC includes electronic stopping, obtained from SRIM [17] calculations as a dumping term. This slowing term depends both on atomic type and velocity. It has a double use. First, it allows to model in a crude way the electronic losses. Second, as it dissipates kinetic energy from the atoms, it allows the calculation to stop when the last cell reaches the kinetic energy threshold. It should be noted that more subtle electronic stopping effects such as electron-phonon coupling are not dealt so that the variation in damage production due to these effects cannot be considered with the present approach.

With the addition of the electronic stopping, PKA energies are then total kinetic energies: ionizing plus ballistic. However, the results presented in the next sections are presented in terms of the corresponding ballistic energies, as deduced from SRIM calculation for the same conditions. Overall, CMDC allows making cascade calculations, which scales linearly with the projectile energy thus ensuring a considerable speed-up compared to regular MD: about 5 orders of magnitude for $1 \mathrm{MeV}$ cascades in iron [16]. See also Section 2.3 for validation of CMDC in the case of $\mathrm{UO}_{2}$. CMDC does not perfectly reproduce MD simulations, namely cascades are known to form nanometric clusters of point defects. Such clusters are poorly described with CMDC. However, we focus in the present work on the total creation of defects and subcascade division. The former is correctly reproduced by CMDC (see Ref. [16] and below) and the same should apply for the latter. Indeed subcascade creation is a high energy process involving projectiles of kinetic energies of at least a few hundred eV. As CMDC describes the kinetics of atoms down to energies below $0.1 \mathrm{eV}$ one can reasonably expect that the subcascade division will be correctly reproduced.

\subsection{Technicalities}

In the present work, uranium dioxide is described using a pair potential of the Buckingham type [18], spherically truncated in real space [19], which had previously been used for cascade simulations $[12,13]$ thus allowing comparison of CMDC results. $\mathrm{Ni}_{3} \mathrm{Al}$ is described with an Embedded Atom Model potential [20]. The potentials have been smoothly connected to the universal Ziegler-Biersack-Littmark (ZBL) potential [17] at short distances.

16 PKA energies following geometrical series from 0.1 to $580 \mathrm{keV}$ were considered, except for U PKA in $\mathrm{UO}_{2}$ where $1 \mathrm{MeV}$ PKA energy is also considered for reasons explained below (Section 3.1). For each atom type and energy, 25 cascades with random initial direction are calculated. As indicated above, PKA energies are total kinetic energies, which correspond to various ballistic energies depending on the type of the PKA (and the material). As an example, Table 1 indicates the ballistic energy corresponding to a $580 \mathrm{keV}$ total energy for the four considered systems and for Fe PKAs in iron. Cells are initialized at room temperature.

Defects are detected in quenched final atomic configurations,
Table 1

Ballistic energy (in keV) corresponding to a total PKA kinetic energy of $580 \mathrm{keV}$.

\begin{tabular}{llllll}
\hline $\mathrm{E}_{\text {tot }}=580 \mathrm{keV}$ & $\mathrm{U}-\mathrm{UO}_{2}$ & $\mathrm{Ni}-\mathrm{Ni}_{3} \mathrm{Al}$ & $\mathrm{Fe}-\mathrm{Fe}$ & $\mathrm{Al}-\mathrm{Ni}_{3} \mathrm{Al}$ & $\mathrm{O}-\mathrm{UO}_{2}$ \\
\hline $\mathrm{E}_{\mathrm{b}}$ & 367 & 307 & 288 & 165 & 82 \\
\hline
\end{tabular}

based on a Wigner Seitz division of the crystal. In the next sections the number of defects refers to the number of created FPs. Present results are compared with Fe cascade in iron previously produced [16] with CMDC and an EAM potential.

\subsection{Validation of cell molecular dynamics for cascade}

The validity of the CMDC results has already been discussed in the case of self PKA in iron [16]. We found that the efficiency calculated with CMDC nicely follows standard MD predictions. Further validation can be extracted from the comparison of the $\mathrm{CMDC}$ results obtained for uranium PKA in $\mathrm{UO}_{2}$ with the ones obtained with standard MD with the same potential [12]. Fig. 1 shows the number of defects predicted by CMDC and standard MD. Two sets of CMDC runs are shown; the first one corresponding to usual CMDC calculations is labelled as "short runs". One can see that for these CMDC short runs the number of defects is consistently larger than the one obtained with MD. In these calculations, the kinetic energy thresholds are such that the cells are turned off after less than $0.5 \mathrm{ps}$.

Standard MD cascade simulations in $\mathrm{UO}_{2}$ [12] have proven that some FPs recombinations take place in the first picoseconds at the end of the cascades. To catch these recombinations we performed additional calculations with a different turning off criterion: each turned on cells remains active for at least 5 ps. These other calculations are termed as "long runs" since the CPU time is then larger. Using such long runs one obtains the set of blue points in Fig. 1. One can see that the long run results are extremely close to the standard MD numbers.

The occurrence of such recombinations of FPs in the few first picoseconds at the end of cascade is especially important in $\mathrm{UO}_{2}$. Indeed it is known that $\mathrm{FP}$ recombinations in $\mathrm{UO}_{2}$ exhibit quite a complex energy landscape with low energy barriers for vacancy-

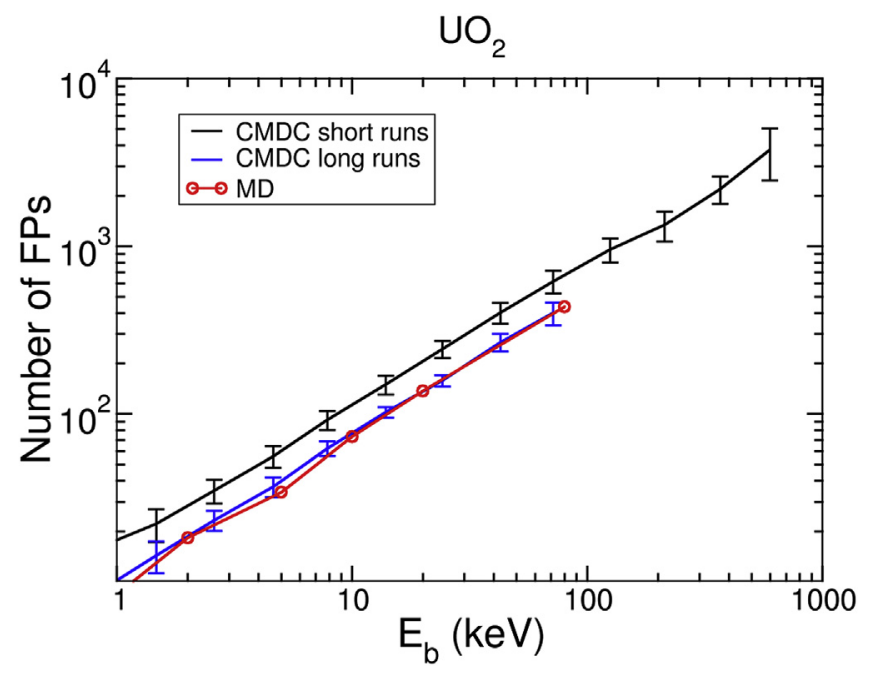

Fig. 1. Defect creation for $U$ PKA in $\mathrm{UO}_{2}$ as a function of ballistic energy. Black and blue lines and error bars are CMDC short and long runs respectively (see text). Red points are standard DM calculations form literature [12] using the same potential. (For interpretation of the references to colour in this figure legend, the reader is referred to the web version of this article). 
interstitial distances of a few nearest neighbors [21]. Such close FPs with low energy barriers for recombination can be labelled as metastable in the sense that their recombination energies are lower than the migration energies of the isolated vacancies and interstitials. One can however note that the ratio between short and long runs is almost perfectly constant and equal to 1.5. Thus one third of the FPs created at the end of the cascades recombines within 5 ps. The other FPs are much longer to recombine. Indeed, regular MD calculations [12] were pursued up to $100 \mathrm{ps}$ with no further recombinations. Note that this metastability effect is specific to $\mathrm{UO}_{2}$. Indeed, for $\mathrm{Ni}_{3} \mathrm{Al}$ the number of defects predicted by short and long runs differ by not more than $10 \%$. Beyond this metastability issue the results of CMDC in terms of created defects are consistent with the ones produced by standard MD. In the following we focus on the short run results. Nonetheless, qualitative conclusions would remain unchanged with long run simulations (see also Section 5).

Finally, as far as CPU times are concerned, one can note that, for uranium PKAs, long runs last on average $40 \%$ more CPU time than the short ones with typical cpu time of $40 \mathrm{~h}$ for $32 \mathrm{keV}$ cascade short runs. For oxygen $32 \mathrm{keV}$ PKA short runs lasts $49 \mathrm{~h}$ and the increase between long and short runs rises to 70\%. The larger gain for O PKAs is due to the fact that O PJKA cascade are less dense (see below Section 4) so that many parts of the crystal are just crossed by the projectiles with little heat transfer. In short runs such cells are readily turned off while in long runs they have to remain active for $5 \mathrm{ps}$.

\subsection{Effective threshold displacement energy}

The NRT formula relies on the displacement energy $\left(E_{d}\right)$. In a mono-elemental solid this quantity can be easily calculated with MD [22]. In alloys, it is equally simple to calculate the displacement energy for each atom type. With the present potential, $E_{d}$ equals $40 \mathrm{eV}$ and $20 \mathrm{eV}$ for uranium and oxygen atoms respectively in $\mathrm{UO}_{2}$ [13]. For $\mathrm{Ni}_{3} \mathrm{Al}$, we calculated $E_{d}$ values of $15 \mathrm{eV}$ and $41 \mathrm{eV}$ for $\mathrm{Ni}$ and $\mathrm{Al}$ atoms respectively for the present potential. From these type dependent displacement energies $\left(E_{d}^{i}\right)$, one should calculate an effective displacement energy ( $\left.E_{d}^{\text {eff }}\right)$ to enter in the NRT formula in order to obtain the number of created defects. Few works exist in the literature dealing with this transformation from type dependent displacement energies to a global effective one. It is generally assumed [23], following Binary Collision Approximation (BCA) calculations in alloys by Ghoniem et al. [24], that the effective energy is obtained with the following formula:

$E_{d}^{\mathrm{eff}}=\frac{1}{\sum_{i} S_{i}\left(E_{d}^{i}\right)^{-1}}$

where $S_{i}$ is the atomic fraction of atoms of type i. This formula amounts to partition the ballistic energy according to the stoichiometry and then applying the NRT formula independently for each species.

With this formula the effective displacement energies for $\mathrm{UO}_{2}$ and $\mathrm{Ni}_{3} \mathrm{Al}$ are $24 \mathrm{eV}$ and $18 \mathrm{eV}$ respectively. In the following, we discuss the number of created defects in terms of efficiency with respect to the NRT law using the Ghoniem formula (Eq. (3)).

\section{Variation of defect production with ballistic energy}

\subsection{Total defect production}

The total numbers of produced FPs as a function of ballistic energies are compared in Fig. 2 with the corresponding NRT prediction. There are some common features to all cases: at low energies the production of defects is sublinear so that the number of created FPs is always lower than the one predicted by the NRT formula for energies larger than $1 \mathrm{keV}$.

The evolution of the number of defects created by Ni PKA in $\mathrm{Ni}_{3} \mathrm{Al}$ and $\mathrm{U}$ in $\mathrm{UO}_{2}$ resembles to what is observed in pure metals $[5,7]$. One can distinguish two steps. First the number of defects is sublinear with energy and varies as a power law with $E_{b}$ :

$N_{\mathrm{FP}}^{C M D C} \sim E_{b}^{0.81}$ for Ni and $N_{\mathrm{FP}}^{C M D C} \sim E_{b}^{0.76}$ for U PKAs.

Then the number of created defects switches to a linear variation with energy. The change from sublinear to linear takes place around $10 \mathrm{keV}$ for Ni PKA in $\mathrm{Ni}_{3} \mathrm{Al}$. For $\mathrm{U}$ PKA the defect production remains sublinear up to much higher energy. Indeed, linearity is observed only for ballistic energies larger than $270 \mathrm{keV}$. We checked this linearity by performing, in this case only, additional cascade calculations at $1 \mathrm{MeV}$ total energies, (i.e. $\mathrm{E}_{\mathrm{b}}=597 \mathrm{keV}$ ).

The production of defects for $\mathrm{Al}$ PKA in $\mathrm{Ni}_{3} \mathrm{Al}$ and $\mathrm{O}$ PKA in $\mathrm{UO}_{2}$ appears to be different. Indeed, for Al PKA the number of FP evolves continuously as a power law with the ballistic energy: $N_{\mathrm{FP}}^{C M D} \sim E_{b}^{0.83}$, with no sign of change to linearity. Whereas, for $\mathrm{O}$ PKA it seems to have two different power law regimes. For $E_{b}<16 \mathrm{keV}, N_{\mathrm{FP}}^{C M D C}$ scales as $E_{b}^{0.91}$; then for $E_{b}>16 \mathrm{keV}$ up to $580 \mathrm{keV}$ (i.e. $E_{b}=82 \mathrm{keV}$ ), $N_{\mathrm{FP}}^{C M D C}$ scales as $E_{b}^{0.54}$, which is even less linear.

\subsection{Applicability of the arc-dpa formula}

The points in Fig. 3 shows the efficiency of production of defects in the four cases considered herein. In all cases one can note a decrease of efficiency with increasing ballistic energy. Beyond this common point noticeable differences appear. In the $U$ and Ni cases the efficiency of defects production tends to become constant for high energies. Conversely, for $\mathrm{Al}$ and O PKAs there is no sign of saturation in the decrease of $\chi$.

As explained above NRT formula assumes a linear defect production with ballistic energy which overestimates the defect production. In view of the weakness of the NRT standard, there have been many discussions about the opportunity to define a better standard. The most recent proposition was made by an OECD expert group [25]. They introduce the so-called "Athermal Recombination Corrected - Displacement Per Atom" (arc-dpa) formula. This formula aims to reproduce the initial decrease then saturation of the efficiency:

$\chi^{a r c-d p a}=c+(1-c)^{*}\left(0.4 \frac{E_{b}}{E_{d}}\right)^{b}$

Hence the number of produced FPs is:

$\left.N_{\mathrm{FP}}^{a r c-d p a}=0.4 \frac{E_{b}}{E_{d}} \quad c+(1-c)^{*}\left(0.4 \frac{E_{b}}{E_{d}}\right)^{b}\right)$

These formulas have proven to reproduce accurately the defect creation in iron and seem promising for other metals. We tried to apply the arc-dpa formulas to our results.

For Ni PKA the data points can be satisfactorily fitted with the arc-dpa formula with effective displacement energy equals to, $\mathrm{E}_{\mathrm{d}}=17.8 \mathrm{eV}$, and parameters: $\mathrm{c}=0,29$ and $\mathrm{b}=-0,34$ (continuous green lines in Fig. 3). In the other cases the efficiency cannot be fitted with the arc-dpa formula. For $\mathrm{U}$ or O PKAs in $\mathrm{UO}_{2}$, the fit is very poor. This can be explained by the efficiencies observed at low energies. Indeed, the number of created FPs is much larger than the one predicted by the NRT law, hence the efficiency exceeds one up to energies of about $1 \mathrm{keV}$. Conversely, the arc-dpa formula relies on the assumption that the efficiency equals one for $E_{b}=E_{d} / 0.4$. In 

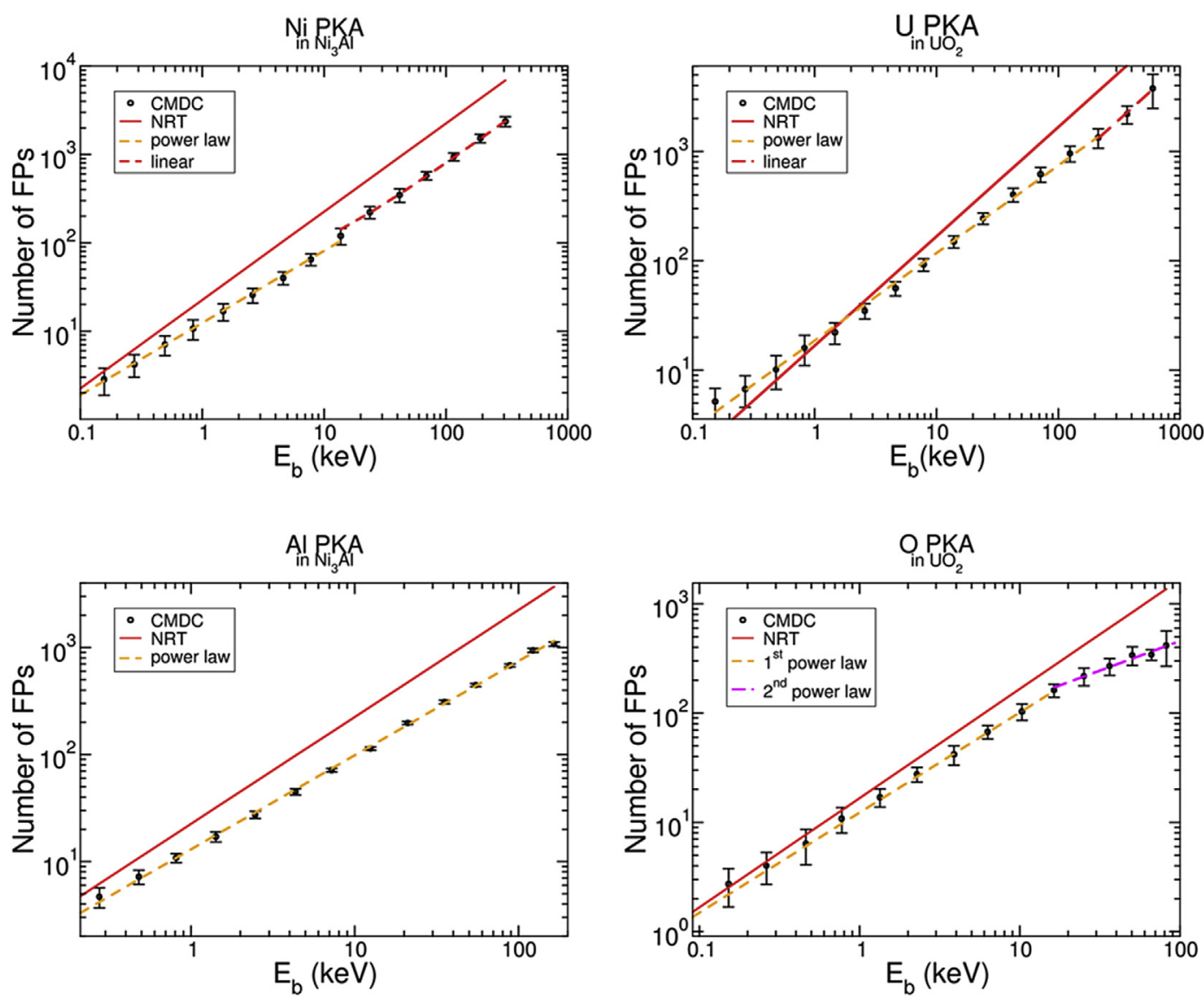

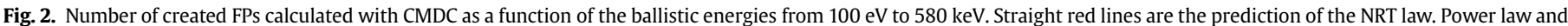
linear fits of parts of the defect curves are shown. (For interpretation of the references to colour in this figure legend, the reader is referred to the web version of this article).

the case of $\mathrm{UO}_{2}$ the efficiency is then expected to be one for $60 \mathrm{eV}$ while it is equal to about 1.6 and 3 for $O$ and $U$ PKAs respectively. Therefore the evolution of $\chi$ cannot be fitted with the arc-dpa formula. For Al PKA in $\mathrm{Ni}_{3} \mathrm{Al}$ the arc-dpa formula performs better. However, it is not perfect. For instance at low energies the calculated efficiency remains lower than one. Moreover, the arc-dpa formula assumes that the efficiency will eventually become constant at large energies, which is not observed in the $\mathrm{Al}$ case.

\section{Subcascade formation and linearity of defect production}

\subsection{Subcascades as dense defect pockets}

The decrease of the efficiency of defect formation with respect to the NRT law has been observed very early in MD calculations $[5,6]$. It has also for long been noticed that this decrease eventually comes to an end in pure metals and the defect production turns to linear with ballistic energy. The common explanation for this high energy linearity of defect production relates to the apparition of subcascades. It was apparently first suggested by Averback et al. [5]: "the phenomenon of saturation of the efficiency can be plausibly explained in terms of subcascade formation." It was indeed verified for various metals (among which $\mathrm{Cu}[5], \mathrm{Ag}^{5}, \mathrm{Fe}^{7}$ ) and was subsequently considered as well established, e.g. Stoller [7] wrote: "the ratio of MD stable displacements divided by the number obtained from the NRT model decreases with energy until subcascade formation becomes prominent".

The present calculations allow us to revisit this stated relationship between subcascade formation and linearity of defect production, i.e. saturation of the efficiency decrease. To do so we need to properly define subcascade formation. Subcascades are in essence the result of dynamical processes, which can be detected in MD simulations following the movements of all displaced atoms [26]. However, it is possible to track their formation analyzing the final state defects. Subcascades appear when the mean free path of the PKA between two collisions is larger than the characteristic length of the defect pocket created by the collision. Observing and counting disconnected dense defect pockets allows characterizing subcascade formation. This is indeed what is done commonly by "eye inspection" of the cascade debris. One then observes dense areas of defects loosely connected with linear tracks involving much less defects. Each of these defect pockets is then attributed to a particular subcascade. To go beyond such qualitative methods, we define a method to dispatch the defects among various dense defect pockets.

The procedure is the following:

1 Defects (both vacancies and interstitials) are linked as neighbors if they are separated by less than some cutoff radius (presently $1.5 \mathrm{~nm}$ );

2 Defects, which have more than a specified number of neighbors (presently 8), are labelled as highly coordinated defects. Such defects and their neighbors belong to the same defect pocket;

3 Defects not connected to highly coordinated defects are labelled as isolated and do not belong to any subcascade.

Applying this procedure, defects are dispatched among different defect pockets except for the isolated ones. An example of such 

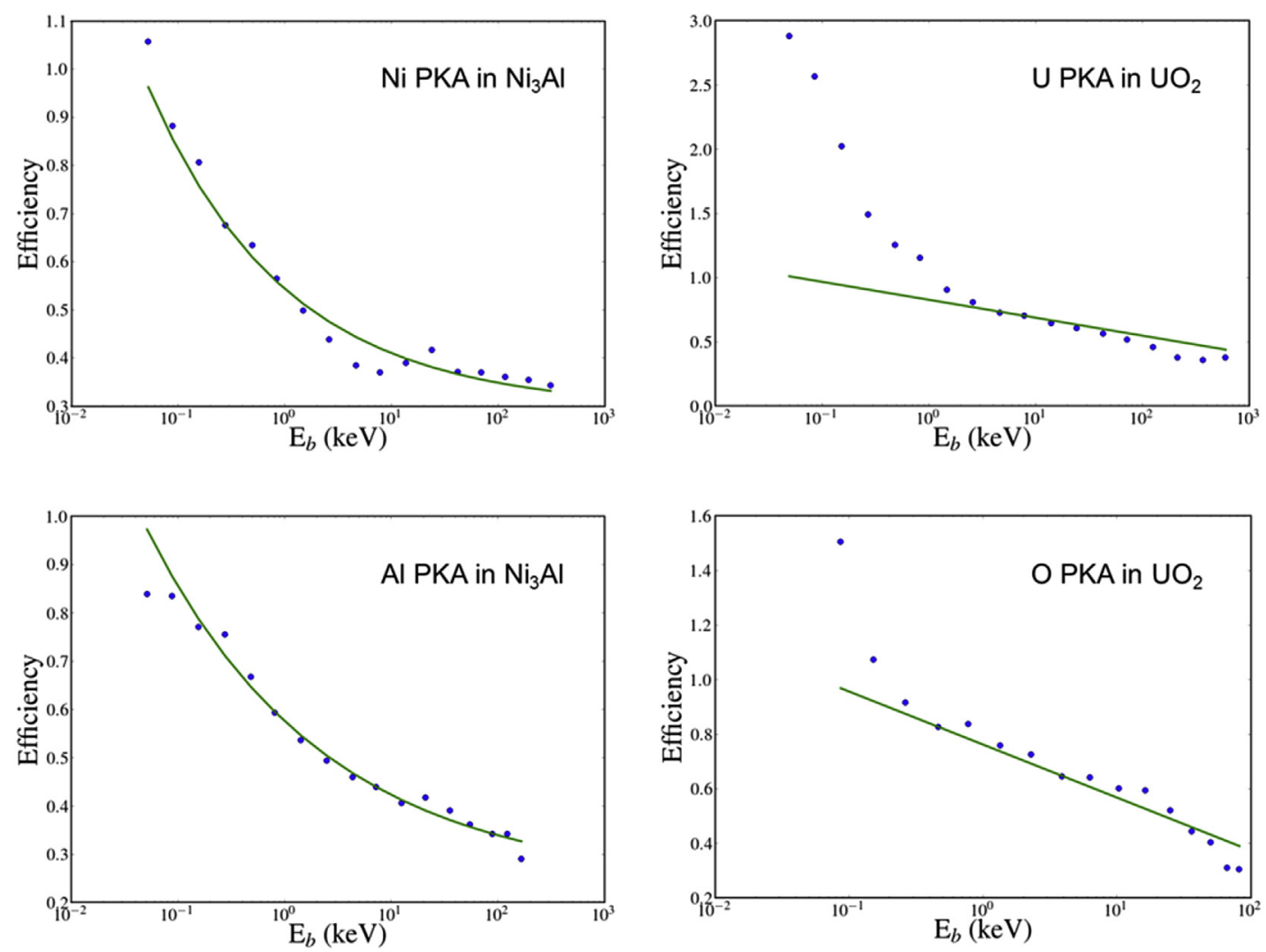

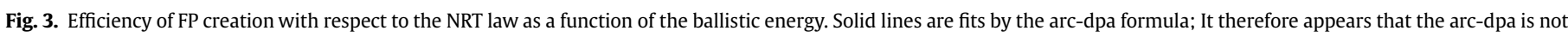

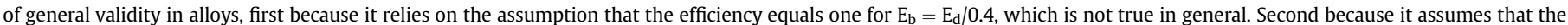
defect production will exhibit a linear feature at high energies, which once again is not always the case.

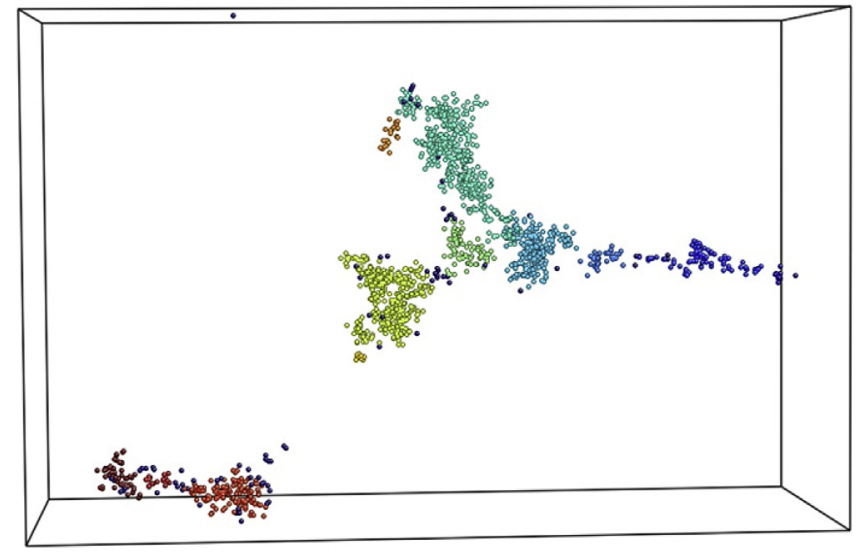

Fig. 4. Division in subcascades for $\mathrm{E}_{\text {tot }}=180 \mathrm{keV}$ Ni PKA in $\mathrm{Ni}_{3} \mathrm{Al}$. Defect pockets are indicated by various colors. Isolated defects are in dark blue. (For interpretation of the references to colour in this figure legend, the reader is referred to the web version of this article).

dispatching is given in Fig. 4.

The above mentioned thresholds for neighbor connection or highly coordinated defects have been chosen to reproduce qualitatively "eye inspection" and to give sensible results for Fe cascades in iron (see below). Changing these thresholds slightly affects the division in subcascades but not the following qualitative discussion.

Defect pockets are then sorted according to their number of defects in decreasing order. The first defect pocket (DP1) is thus the one containing the most number of defects, irrespective of whether it appears at the beginning or at the end of the cascade. Subsequently, DP2 relates to the second, DP3 to the third and so on. As mentioned before, these defect pockets are considered to be the result of disconnected subcascades.

Fig. 5 illustrates the seminal iron case. It is based on the cascade calculations presented in a previous paper [16]. Left panel shows the number of defects as a function of ballistic energy, obtained with CMDC, as well as the number of defects in DP1, DP2, DP3 and isolated defects. For low energies the number of defects in DP1 follows the total number of defects with a small portion of isolated defects. In this energy range there is no subcascade formation. Above $40 \mathrm{keV}$, the number of defects in DP1 saturates while the numbers in DP2 and DP3 rise. This is the indication of subcascade formation. At $100 \mathrm{keV}$, DP2 has reached saturation. One clearly observes the formation of subcascades, which multiply for larger energies.

The relative amount of defects either isolated or contained in each subcascades is indicated in the right panel in Fig. 5. It can be interpreted along the same lines as the left panel of the same figure. Below $40 \mathrm{keV}, 60-80 \%$ of defects are in DP1 and $20 \%$ are isolated; there is no subcascade formation. Beyond $40 \mathrm{keV}$, the proportion of defects in DP1 decreases steadily, as its size saturates while more and more defects are created. The proportion of defects in DP2 and DP3 then rises before saturating and decreasing since their own size saturates. The proportion of isolated defects remains constant. At the highest energy $\left(E_{b}=300 \mathrm{keV}\right), 60 \%$ of the defects are either isolated or contained in DP1, DP2, and DP3, which means that additional subcascades (DP4, DP5, etc.) contain the rest of the defects.

One observes that the onset of subcascade formation takes place in the same energy range as the saturation of the efficiency decrease of defect production (around $40 \mathrm{keV}$ ). The connection 

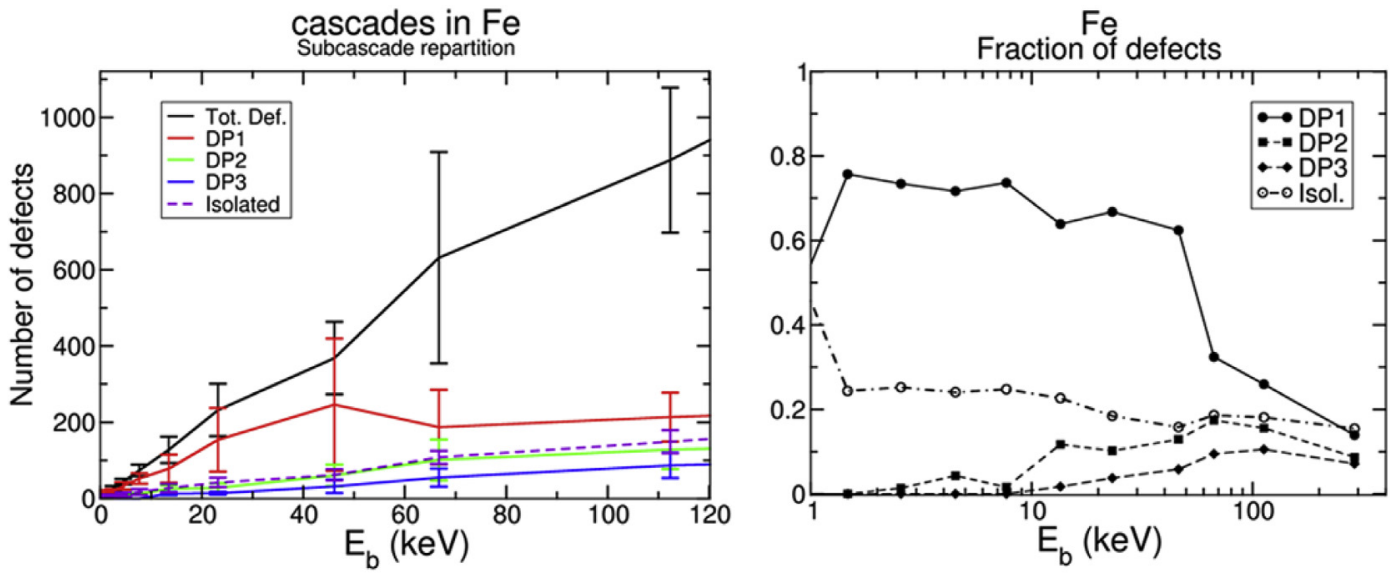

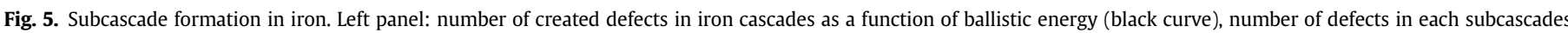

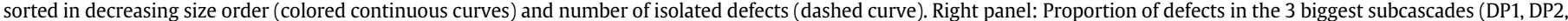
DP3) and isolated defects. (For interpretation of the references to colour in this figure legend, the reader is referred to the web version of this article).

between subcascade formation and linearity of defect formation is reproduced.

\subsection{Case of PKAs in alloys}

The same procedure of dispatching defects in defect pockets has been applied to our calculations on $\mathrm{UO}_{2}$ and $\mathrm{Ni}_{3} \mathrm{Al}$. The proportions of defects in the three biggest defect pockets are given in Fig. 6. The shapes of the curves are close to what was observed in iron. Initially most of defects are in the biggest pocket. Then, at some energy the proportion of defects in the biggest pocket falls while other defect pockets appear and start to contain a large number of defects. One can estimate that subcascades appear around this energy where the biggest defect pocket contains only $50 \%$ of the total number of
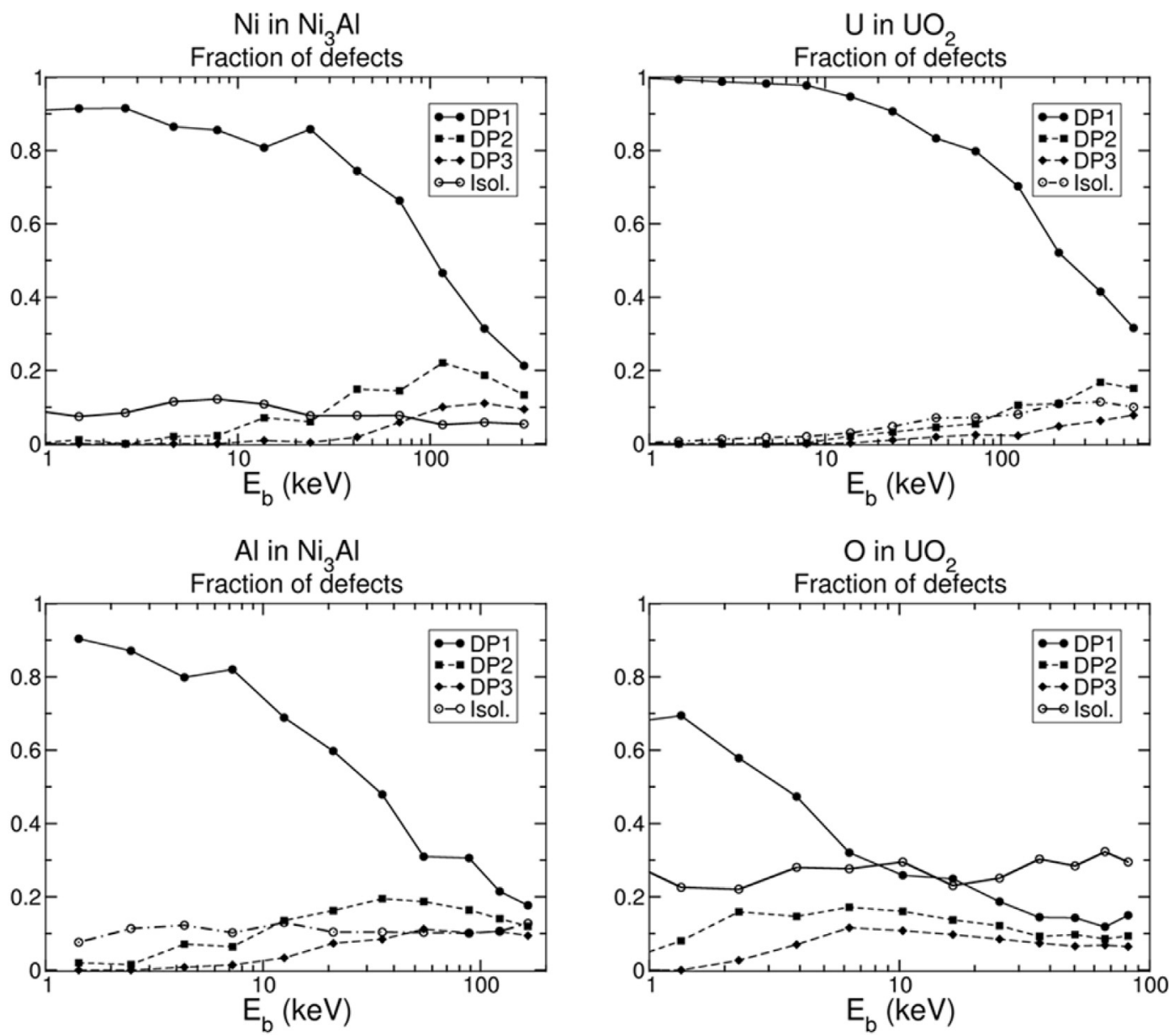

Fig. 6. Subcascade formation for $\mathrm{Ni}$ and $\mathrm{Al}$ in $\mathrm{Ni}_{3} \mathrm{Al}$ and $\mathrm{U}$ and $\mathrm{O}$ in $\mathrm{UO}_{2}$. Proportion of defects in the 3 biggest subcascades (DP1, DP2, DP3) and isolated defects. 

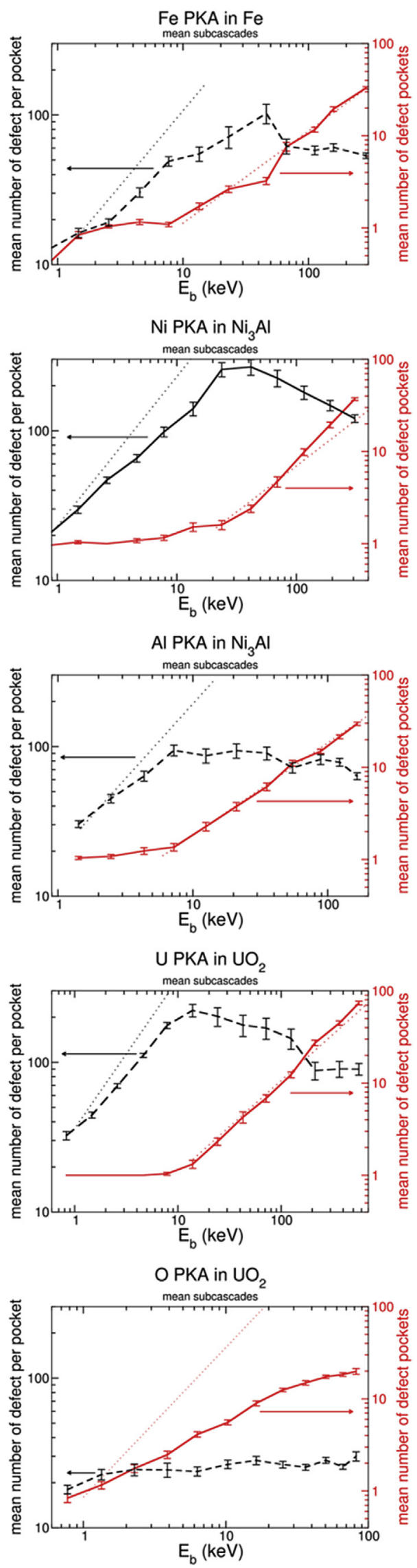

defects.

With this approximate threshold in mind one can note that for $U$ in $\mathrm{UO}_{2}$ the onset of subcascade formation (a little more than $200 \mathrm{keV}$ ) is close to the energy of saturation of the efficiency of defect formation. Conversely, for $\mathrm{Ni} \mathrm{PKA}$ in $\mathrm{Ni}_{3} \mathrm{Al}$ the energy of subcascade formation appears to be larger (around $100 \mathrm{keV}$ ) than the one of linearity (about $10 \mathrm{keV}$ ). Furthermore, for $\mathrm{Al}$ and $\mathrm{O}$, the division in subcascade appears while linearity of defect production is never reached. This is especially striking for $\mathrm{O} \mathrm{PKA}$ in $\mathrm{UO}_{2}$ where subcascades appear at very low energy, as early as $3 \mathrm{keV}$ and no sign of saturation of efficiency decrease can ever be seen up to $\mathrm{E}_{\mathrm{b}}=80 \mathrm{keV}$.

Therefore, one has to state that, in the general case, the linearity of defect production and subcascade formation do not necessarily take place at the same energy; thus invalidating the traditional link between the two phenomena.

\section{Average numbers of subcascades and defect per subcascades}

As we performed more than just a few cascade calculations, we were able to make statistical averaging even at the highest energies. We use the same procedure of defect dispatching in various defect pockets. From this post-processing, we obtain, for each PKA energy, the distribution of the number of defect pockets and the distribution of the number of defects in the defect pockets. Averaging these two distributions yields to the average number of defect pockets, $\tilde{N}_{S C}\left(E_{b}\right)$, and the average number of defects in the defect pockets, $\tilde{N}_{S C}^{\text {def. }}\left(E_{b}\right)$.

The total number of defects can then be estimated as:

$N_{\mathrm{FP}}^{C M D C}=\tilde{N}_{S C}\left(E_{b}\right) \times \tilde{N}_{S C}^{\text {def. }}\left(E_{b}\right)+\tilde{N}_{\text {isol }}^{\text {def }}\left(E_{b}\right)$

where $\tilde{N}_{\text {isol }}^{\text {def }}\left(E_{b}\right)$ is the number of isolated defects.

The number of isolated defects proves to vary linearly with ballistic energy (see Fig. 5 for iron). The linearity or none linearity of defect production, therefore, lies in the variations with ballistic energy of $\tilde{N}_{S C}\left(E_{b}\right)$ and $\tilde{N}_{S C}^{\text {def. }}\left(E_{b}\right)$. The values of these two quantities as a function of ballistic energies in the different cases are given Fig. 7.

One can see that, as expected, the average number of subcascades $\left(\tilde{N}_{S C}\left(E_{b}\right)\right)$ equals one up to a certain threshold where it starts to rise. For ballistic energies lower than this threshold, $\tilde{N}_{S C}^{\text {def. }}\left(E_{b}\right)$ is simply the number of defects in the only existing defect pocket. It increases sublinearly with the ballistic energy. Thus the efficiency of defect production with respect the NRT law is decreasing at such low energies.

For energies larger than the onset of subcascade formation, the evolutions of $\tilde{N}_{S C}\left(E_{b}\right)$ and $\tilde{N}_{S C}^{\text {def. }}\left(E_{b}\right)$ show a rather unexpected variety of behaviors. For iron and uranium in $\mathrm{UO}_{2}$, the number of defect pockets starts to exceed one then varies linearly with $E_{b}$. Concurrently, the number of defects per pocket stops rising, then decreases to eventually saturate to a roughly constant value. From this energy on, Eq. (6) shows that the defect creation is linear with ballistic energy. Indeed $\tilde{N}_{S C}^{\text {def. }}\left(E_{b}\right)$ being constant and $\tilde{N}_{S C}\left(E_{b}\right)$ being linear with energy, their product varies linearly with energy.

The situation appears more complex for $\mathrm{Ni}$ PKA in $\mathrm{Ni}_{3} \mathrm{Al}$. Indeed, after its initial rise, the number of defects per pocket seems to continuously decrease even at the largest energies. Simultaneously, the number of defect pockets increases more than linearly with

Fig. 7. Average number of defect pockets (right scale) and average number of defects per pocket (left scale) as a function of ballistic energy. The dotted lines are linear guide for the eyes. 
energy. Thus, the observed linear production of defect in this case results from concurrent decrease of $\tilde{N}_{S C}^{\text {def. }}\left(E_{b}\right)$ and overlinear increase of $\tilde{N}_{S C}\left(E_{b}\right)$ with their product being constant. It appears then that the linearity of defect production for these cascades does not have the same origin as the ones observed in iron and for U PKA in $\mathrm{UO}_{2}$.

As far as the $\mathrm{Al}$ in $\mathrm{Ni}_{3} \mathrm{Al}$ and $\mathrm{O}$ in $\mathrm{UO}_{2}$ cases are concerned, one observes a sublinear production of defects at all energies with no sign of saturation for the efficiency. However, the origins of this apparently identical behavior seem to be different in both cases.

For Al PKA the number of defect pockets appears linear with energy while the number of defects in the pockets appears to be slowly decreasing with energy. The product is therefore sublinear with energy. Conversely, $\mathrm{O}$ PKAs in $\mathrm{UO}_{2}$ create pockets of constant number of defects but the number of defect pockets increases sublinearly with energy. The continuous decrease of the efficiency therefore lies in this sublineatity of the number of defect pockets.

Additionally, we checked in the case of U PKA that long runs (see Section 2.3) lead qualitatively to the same results than short runs. The evolutions of $\tilde{N}_{S C}^{\text {def. }}\left(E_{b}\right)$ and $\tilde{N}_{S C}\left(E_{b}\right)$ are very similar between long and short runs, up to the maximum energy of long run calculations $\left(E_{b}=72 \mathrm{keV}\right)$. In fact, rescaling the energy scale of short runs by a factor 1.7 leads to results very close to the ones of long runs in terms of $\tilde{N}_{S C}\left(E_{b}\right)$, while $\tilde{N}_{S C}^{\text {def. }}\left(E_{b}\right)$ is consistently smaller for long runs than for short runs. The difference between long and short runs directly comes from the lesser defect production in long runs. This explains naturally why $\tilde{N}_{S C}^{\text {def. }}\left(E_{b}\right)$ is smaller for long runs. It also leads to a shift in energy for the evolution of $\tilde{N}_{S C}\left(E_{b}\right)$ as the smallest assemblies of defects (beyond 8 defects) are not counted as defect pockets but as isolated defects, so that a larger ballistic energy is needed in long runs to have the same $\tilde{N}_{S C}^{\text {def. }}\left(E_{b}\right)$ than in short runs. Nevertheless the results of long and short runs remain qualitatively the same and their differences do not affect the discussion. Note that as far as $\mathrm{Ni}_{3} \mathrm{Al}$ is concerned, defect production differs only by $10 \%$ between the two types of run, so that short and long runs results should be very close in terms of average defect pockets.

Our results allow us to discuss the linearity of defect production in high energy cascades. The common view is implicitly based on the assumption that above some energy the subcascades reach a constant size (on average) while their number is linear with energy. These assumptions have been proven in the BCA framework through fractal analyses [27]. But this study was restricted to monoelemental solids and the calculations did not take into account inelastic energy loss so that their validity for complete (elastic plus inelastic) energy loss is not warranted.

Our calculations show that this simple picture is not of general validity in alloys. While it proves correct for cascades in iron and for $\mathrm{U}$ PKA is $\mathrm{UO}_{2}$, it appears that the number of subcascades at high energies can be over or sublinear with ballistic energy. Meanwhile, the number of defects per pocket does not always reach a constant value. This alloy effect certainly pertains to the mass difference between the constituents of the alloys. It should therefore be less important in alloys made of components of comparable masses.

\section{Conclusions}

Most of cascade calculations have been performed in monoelemental solids. This is especially true for the recent calculations interested in defect production at high PKA energies. Our calculations show that some of the conclusions drawn from these monoelemental studies are in fact only valid in these cases and must be revisited in the case of alloys.

We perform MD to make a systematic study of cascades created by heavy and light constituents in two examples of ordered alloys. Using CMDC we are able to reach quite large energies (up to $580 \mathrm{keV}$ for total kinetic energy). We find that the efficiency of defect creation with respect the NRT formula decreases at intermediate energies, but unlike what is observed in elemental solids it does not always turn to a constant value at high energy. For light PKA in alloys, defect creation remains under linear in ballistic energy at high energy.

Analyzing cascade tracks, we find that the common knowledge equivalence of linearity of defect production and apparition of subcascades does not hold in general for alloys. Subcascades appear very early for PKA's of the light constituent while linearity of defect production is never reached. We calculate the average number of subcascades and average number of defects per subcascades as a function of ballistic energy. We find an unexpected variety of behaviors for these two average quantities above the threshold for subcascade formation.

\section{Acknowledgments}

This work has been performed in the framework of the Primary Radiation Damage Cross Sections Coordinated research Project of the International Atomic Energy Agency.

Thomas Jourdan and Alain Barbu (CEA Saclay) are acknowledged for fruitful discussions.

\section{References}

[1] G.H. Kinchin, R.S. Pease, Rep. Prog. Phys. 18 (1955) 1-51.

[2] M. Norgett, M.T. Robinson, I. Torrens, Nucl. Eng. Des. 33 (1975) 50-54.

[3] ASTM Standard E693-01, 2012.

[4] L.E. Rehn, P.R. Okamoto, Mater. Sci. Forum 15-18 (1987) 985-1001.

[5] R. Averback, R. Benedek, K.L. Merkle, Phys. Rev. B 18 (1978) 4156.

[6] M.W. Guinan, J.H. Kinney, J. Nucl. Mater. 104 (1981) 1319-1323.

[7] R.E. Stoller, J. Nucl. Mater. 276 (2000) 22-32.

[8] R.E. Stoller, in: Comprehensive Nuclear Materials, Elsevier, Oxford, 2012, pp. $293-332$.

[9] K. Nordlund, J. Nord, J. Keinonen, Nucl. Instrum. Methods Phys. B 175-177 (2001) 31-35.

[10] C. Bjorkas, K. Vortler, K. Nordlund, Phys. Rev. B 74 (2006) 140103.

[11] N.V. Doan, R. Vascon, Nucl. Instr. Meth. B 135 (1998) 207-213.

[12] L. Van Brutzel, M. Rarivomanantsoa, D. Ghaleb, J. Nucl. Mater. 354 (2006) $28-35$.

[13] L. Van Brutzel, J.M. Delaye, D. Ghaleb, M. Rarivomanantsoa, Philos. Mag. 83 (2003) 4083-4101.

[14] J. Nord, K. Nordlund, J. Keinonen, Phys, Rev, B 68 (2003) 184104.

[15] N.V. Doan, R. Vascon, Radiat. Eff. Defects Solids 141 (1997) 363-373.

[16] J.-P. Crocombette, T. Jourdan, Nucl. Instrum. Methods Phys. B 352 (2015) $9-13$.

[17] J.F. Ziegler, J.P. Biersack, M.D. Ziegler, SRIM - The Stopping and Range of Ions in Matter Ion Implantation Press, 2008.

[18] N. Morelon, D. Ghaleb, J. Delaye, L. Van Brutzel, Philos. Mag. 83 (2003) $1533-1550$.

[19] D. Wolf, P. Keblinski, S.R. Phillpot, J. Eggebrecht, J. Chem. Phys. 110 (1999) $8254-8282$

[20] G.P. Purja Pun, Y. Mishin, Philos. Mag. 89 (2009) 3245-3267.

[21] L. Van Brutzel, A. Chartier, J.P. Crocombette, Phys. Rev. B 78 (2008) 024111.

[22] J.P. Crocombette, in: S. Yip (Ed.), Handbook of Materials Modeling, Springer, Berlin, 2005, p. 987.

[23] S.J. Zinkle, C. Kinoshita, J. Nucl. Mater. 251 (1997) 200-217.

[24] N.M. Ghoniem, S.P. Chou, J. Nucl. Mater. 155-157 (1988) 1263-1267.

[25] Nordlund, K., Sand, A. E., Granberg, F., Zinkle, S. J., Stoller, R. E., Averback, R. S., Suzudo, T., Malerba, L., Banhart, F., Weber, W. J., Willaime, F., Dudarev, S. L., and Simeone, D., (OECD-NEA, 2015).

[26] A.F. Calder, D.J. Bacon, A.V. Barashev, Y.N. Osetsky, Philos. Mag. 90 (2010) 863-884.

[27] L. Luneville, D. Simeone, W.J. Weber, J. Nucl. Mater. 415 (2011) 55-60. 